\title{
A Rare Case of Flexor Hallucis Longus Tendinitis Due to Sesamoid Bone at the Interphalangeal Joint of the Great Toe: A Case Report
}

\author{
Rajiv Shah ${ }^{1}$, Gurunath S Wachche ${ }^{2}$, Dayanidhi N Desai ${ }^{3}$
}

\begin{abstract}
Aim: This study aims to describe the rare case of flexor hallucis longus (FHL) tendinitis due to the sesamoid bone at the interphalangeal joint of the great toe.

Background: Sesamoids are the intertendinous bone, which is rarely found at the hallucal interphalangeal joint. Occasionally, these bones may be associated with pain syndromes. A high index of suspicion should be exercised for the diagnosis. Detailed clinical examination and sound evaluation of radiology will help to establish the diagnosis.

Case description: Forty-five-year-old male patient presented with pain in the great toe of the left foot and the inability to weight-bear over the forefoot for three years. He could not use the closed footwear because of fixed dorsiflexion at the first interphalangeal joint with severe pain. The patient was diagnosed to have an sesamoid bone in the tendon of FHL at the level of the interphalangeal joint. Excision of sesamoid bone cured him.

Conclusion: The accessory bones are commonly treated conservatively, but those causing tendinitis and chronic pain should be treated surgically with excision.

Clinical significance: The sesamoid bone in tendon of $\mathrm{FHL}$ can lead to tendinitis causing chronic pain and difficulty in walking. A high index of suspicion can only help in early diagnosis.

Keywords: Flexor hallucis longus, Great toe, Interphalangeal joint, Sesamoid, Sesamoid bone, Tendinitis.

Journal of Foot and Ankle Surgery (Asia Pacific) (2020): 10.5005/jp-journals-10040-1123
\end{abstract}

\section{BACKGROUND}

The term sesamoid comes from the Arabic word Semsem, meaning sesame seed. Galen named these small rounded bones in reference to their similarity to sesame seeds. ${ }^{1}$ Sesamoids occurs in the substance of the tendon as either completely or partially ossified or cartilaginous bodies. The degree of ossification is responsible for their radiological presence or absence and the appearance of bipartism. Sesamoids of the foot ossify quite late, but the exact time is unclear. ${ }^{2}$

Sesamoids have a protective function as they reduce the friction between the tendon and other rigid structures, producing a more efficient gliding mechanism between adjacent tissues. ${ }^{3}$ Most of the sesamoids are treated conservatively, but those which are troublesome require a surgical intervention. We present a rare case of the symptomatic sesamoid bone in the tendon of flexor halluces longus at the interphalangeal joint of the great toe, which was surgically managed.

\section{Case Description}

A 45-year-old male patient presented with pain at the great toe of left foot and difficulty in weight-bearing over the forefoot for 3 years. The pain was dull aching, insidious in onset, gradually progressive, and moderate-to-severe. The most striking complaint was a deformity of the great toe in the form of toe remaining off the floor. The patient also had difficulty in walking, and he could walk without keeping the forefoot on the ground. The patient could not wear closed footwear from the last 2 years because of severe pain. No history of trauma or any other disease was positive. The patient
${ }^{1}$ Sunshine Global Hospitals, Vadodara-Bharuch-Surat, Gujarat, India

${ }^{2}$ Department of Orthopedics, Wachche Hospital, Solapur, Maharashtra, India

${ }^{3}$ Department of Orthopedics, Pawan Advanced Orthopedic Centre, Surat, Gujarat, India

Corresponding Author: Gurunath S Wachche, Department of Orthopedics, Wachche Hospital, Solapur, Maharashtra, India, Phone: +91 9822195087, e-mail: wgurunathan@yahoo.com

How to cite this article: Shah R, Wachche GS, Desai DN. A Rare Case of Flexor Hallucis Longus Tendinitis Due to Sesamoid Bone at the Interphalangeal Joint of the Great Toe: A Case Report. J Foot Ankle Surg (Asia Pacific) 2020;7(2):70-72.

Source of support: Nil

Conflict of interest: None

underwent multiple consultations over the past 3 years and was on oral pain killers. Multiple sessions of physiotherapy, together with two local steroid injections, did not help him.

On physical examination of the left great toe, marked tenderness was present over the plantar aspect of the interphalangeal (IP) joint. Toe was in fixed dorsiflexion posture at the IP joint without the possibility of passive movements. There was no instability. Depigmentation of the skin due to prior steroid injections was seen around the medial and plantar aspect of the great toe. The prior digital radiographs of the left great toe in both anteroposterior and oblique view, which were earlier passed as normal (Fig. 1), did show the presence of the sesamoid bone in the plantar aspect of 


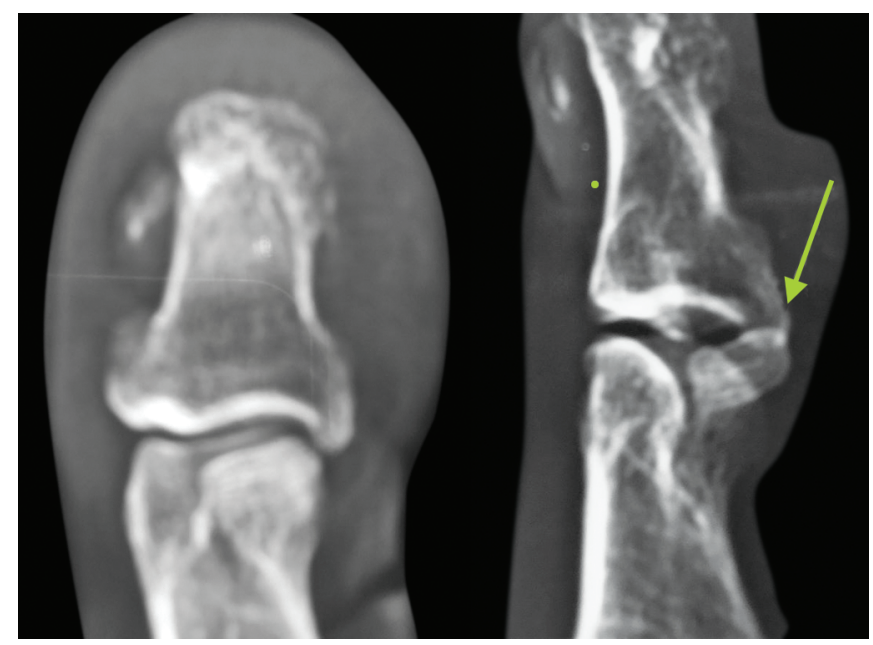

Fig. 1: Plain radiograph of the great toe in anteroposterior and an oblique view showing the presence of a sesamoid bone at the plantar aspect of the interphalangeal joint as marked by an arrow

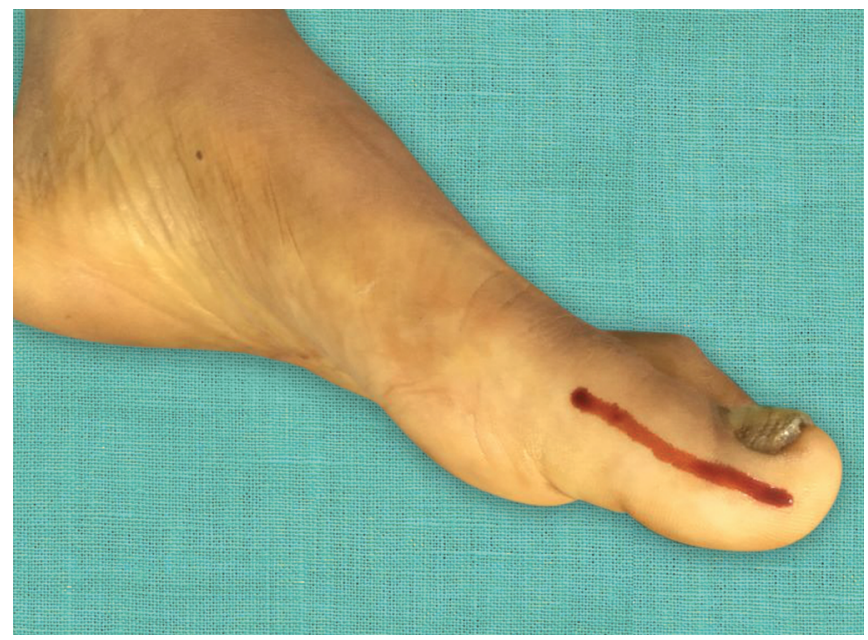

Fig. 3: Skin incision marking for the incision

the IP joint of the great toe. The patient was further evaluated with magnetic resonance imaging (Fig. 2) and diagnosed to have FHL tendinitis caused by the sesamoid bone.

The surgical excision of the sesamoid bone was planned under all aseptic precautions. The patient was positioned in the supine position with two sterile pillows under the operative limb, to get unaided access to the surgical field. A thigh tourniquet was inflated. A longitudinal incision was taken over the medial border of the IP joint of the great toe (Fig. 3). Subcutaneous tissue was dissected, the digital nerve was identified and protected, and FHL tendon along with the sesamoid bone was located (Fig. 4). The sesamoid bone was removed without any injury to tendon (Fig. 5), and the free gliding of tendon FHL was checked. The wound was closed in layers.

A compressive dressing was applied. Sutures were removed on the 10th postoperative day. The postoperative recovery was uneventful. The patient was advised heel-walking for 2 weeks, followed by progressive weight-bearing as tolerated. The patient was followed up for 15 months and had no problems.

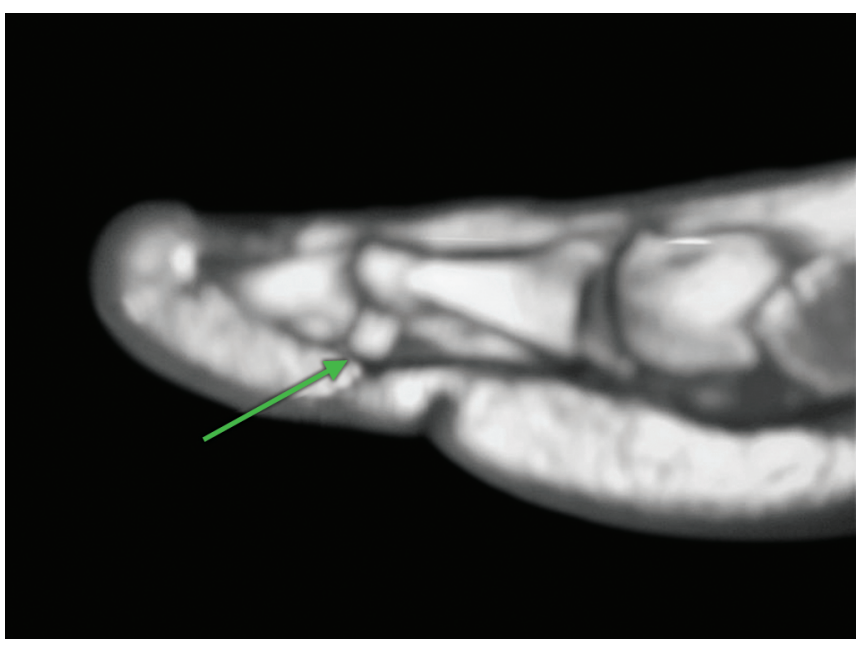

Fig. 2: Magnetic resonance imaging showing sesamoid bone in flexor hallucis longus tendon. Arrow shows the sesamoid bone. Arrows point towards the sesamoid bone

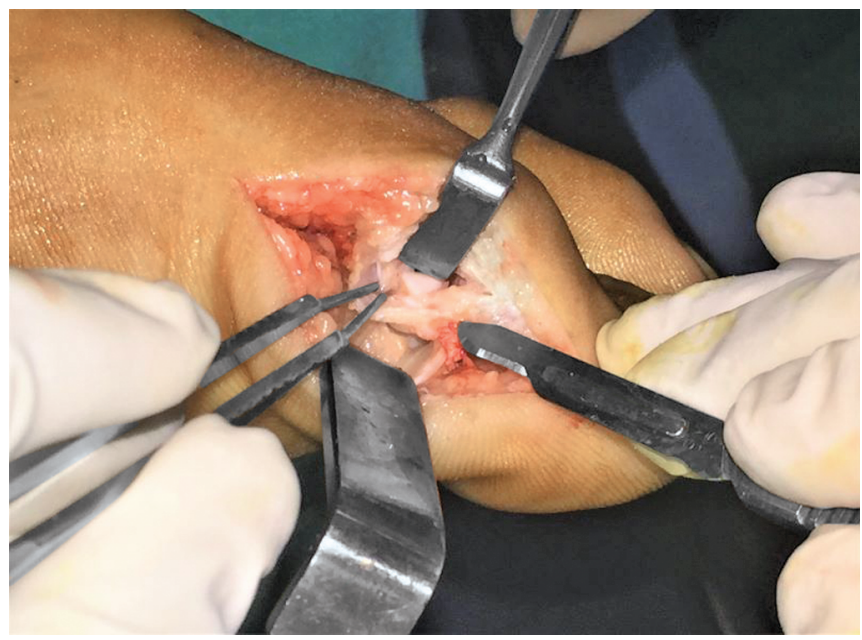

Fig. 4: Sesamoid bone in tendon of flexor hallucis longus

\section{Discussion}

Sesamoids of the foot and ankle develop from their ossification centers and are round or oval. Sesamoids of the foot can be subdivided into hallucal, interphalangeal joint, and less metatarsal sesamoids. Sesamoid bones of the first metatarsophalangeal joint are considered to be a normal part of the skeleton (medial and lateral); however, sesamoid bones at the less metatarsals and the hallux interphalangeal joint are rarely seen and are accepted as accessory bones. ${ }^{3,4}$

The sesamoid bone located at the great toe interphalangeal joint (subhallucal sesamoid), when present, is rare and found in approximately $13 \%$ of the population. Subhallucal sesamoids are often bilateral and are embedded within the tendon of the flexor hallucis longus. ${ }^{5}$ The presence of an ossified sesamoid at the interphalangeal joint may alter biomechanics and limit motion in the joint. ${ }^{6}$ The hallucal interphalangeal sesamoid is an osseous structure associated with the plantar aspect of the interphalangeal joint of the hallux. The dorsal surface 

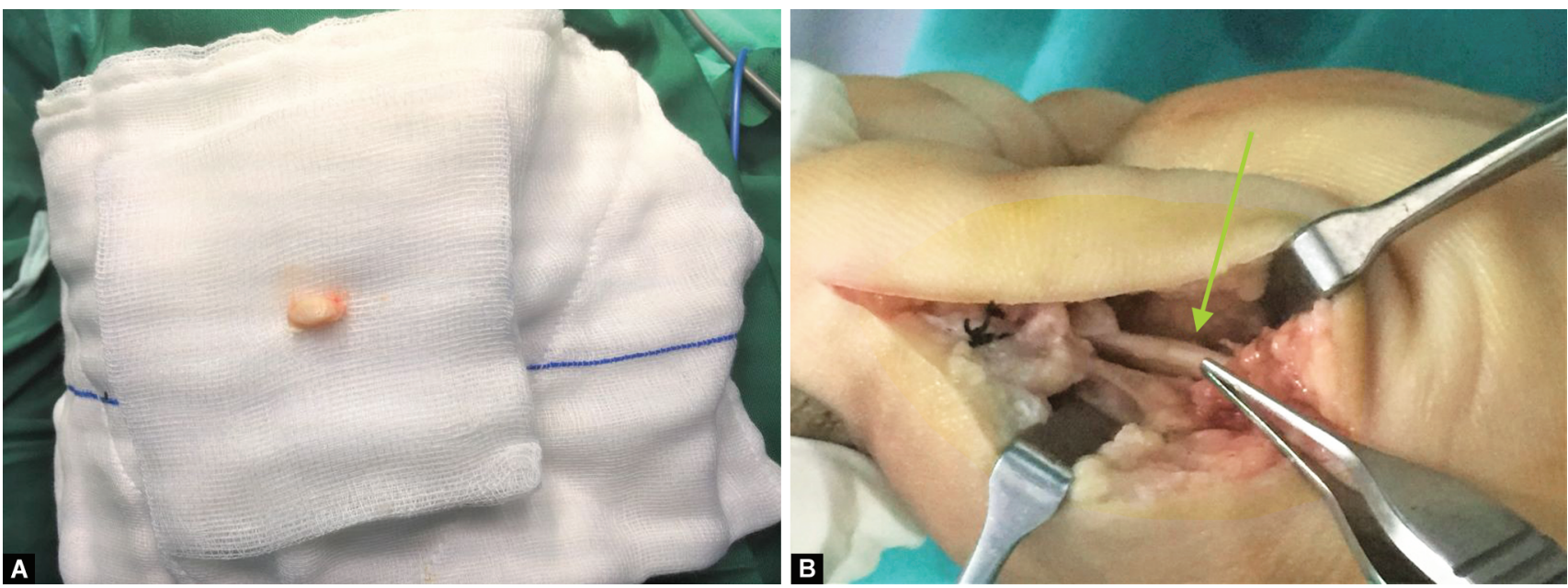

Figs 5A and B: Accessory bone dissected out from the flexor hallucis longus tendon. Tip of the forceps and arrow both are pointing toward the tendon of flexor hallucis longus

of the hallucal interphalangeal sesamoid is predominantly cartilaginous and articulates with the head of a proximal phalanx of the hallux. ${ }^{7}$

Usually, the sesamoids are asymptomatic. Symptomatic sesamoids require a high index of suspicion for early diagnosis. An interphalangeal sesamoid at the great toe is known to cause a decrease in the range of motion at the first metatarsophalangeal joint, with consequent hyperextension of the interphalangeal joint of the hallux. ${ }^{8,9}$ An interphalangeal sesamoid may become troublesome causing painful plantar callosities, inflammation of ossicles, inflammation of FHL tendon, infection, osteonecrosis, and degeneration. ${ }^{10}$ Magnetic resonance imaging and even ultrasonography or nuclear medicine techniques can be helpful in establishing the diagnosis of difficult cases and evaluating foot pain secondary to accessory ossicles. ${ }^{4}$

Once diagnosed, symptomatic sesamoids are treated conservatively. Surgical resection of the accessory bone is indicated in the failed conservative treatment and in symptomatic patients due to irritating surrounding soft tissues or neurovascular structures. We report this case, which is rarely described in the literature; hence, it is commonly missed by clinicians.

\section{Conclusion}

We conclude that most of the accessory ossicles are treated conservatively, but those present at the hallux interphalangeal joint causing chronic pain and difficulty in walking should be treated surgically.

\section{Clinical Significance}

A high index of suspicion helps in the early diagnosis of rare accessory bones in the foot and ankle.

\section{References}

1. Sims AL, Kurup HV. Painful sesamoid of the great toe. World J Orthop 2014;5(2):146-150. DOI: 10.5312/wjo.v5.i2.146.

2. Anwar R, Anjum SN. Sesamoids of foot. Curr Orthop 2005;19(1):40-48. DOI: 10.1016/j.cuor.2005.01.001.

3. Ergun S, Saygi. B. Accessory lesser metatarsal sesmoids in all of the metatarsophalangeal joint. J Am Podiatr Med Assoc 2017;107(3): 223-225. DOI: 10.7547/15-195.

4. Kumar Vora BM, Steven Wong BS. Common accessory ossicles of the foot: Imaging features, pitfalls and associated pathology. Singapore Med J 2018;59(4):183-189. DOI: 10.11622/smedj.2018046.

5. Banerjee R, Bradley MP. Clinical pearls: locked great toe. Acad Emerg Med 2003;10(8):878-880. DOI: 10.1197/aemj.10.8.878.

6. Kenechi Nwawka O, Hayashi D. Sesamoids and accessory ossicles of the foot: anatomical variability and related pathology. Insights Imaging 2013;4(5):581-593. DOI: 10.1007/s13244-013-0277-1.

7. Roukis TS, Hurless J. The hallucal interphalangeal sesamoid. J Foot Ankle Surg 1996;35(4):303-308. DOI: 10.1016/s1067-2516(96)80078-0.

8. Kumar S, Rajagopal K. Subhallucal interphalangeal sesamoiditis. J Clin Diagnos Res 2015;9(5):TD01-TD02. DOI: 10.7860/ JCDR/2015/13210.5870.

9. Wakhlu A. An uncommon cause of great toe pain: sesamoiditis. J Indian Rheumatol Assoc 2004;12.

10. Chang SH, Matsumoto T. Stenosing tenosynovitis of the flexor hallucis tendon associated with the plantar capsular accessory ossicles at the interphalangeal joint of great toe. Case Rep Orthop 2017;2017:2146762. DOI: 10.1155/2017/2146762. 